\title{
ВРЕМЕННАЯ ПЕРСПЕКТИВА В УСЛОВИЯХ НЕГАРАНТИРОВАННОЙ РАБОТЫ
}

\author{
Татьяна С. Чуйкова ${ }^{1 *}$, Дарья И. Сотникова ${ }^{2}$ \\ ${ }^{1}$ Башкирский государственный педагогический университет \\ им. М. Акмуллы, г. Уфа, Российская Федерачия \\ 2 ПАО «БАНК УРАЛСИБ», г. Уфа, Российская Федерачия \\ *E-mail: cts1004@bspu.ru
}

Исследование выполнено при финансовой поддержке РГНФ и АН РБ, проект № 15-16-02017 «Отношение к работе в условиях негарантированной занятости»

Статья посвящена изучению особенностей временной перспективы у сотрудников, находящихся в условиях негарантированной работы. Авторы, отталкиваясь от результатов исследований, фиксирующих искажение временной перспективы у безработных, предположили, что сходные эффекты могут наблюдаться в ситуации негарантированной работы. Анализ исследований временной перспективы, представленный в статье, показал отсутствие подобных исследований, в то же время специфика переживаний в ситуации негарантированной работы, связанных с неопределенностью настоящего и будущего, предполагает изменение временной перспективы в качестве одного из своих следствий. В качестве другого не менее важного следствия переживания негарантированной работы в исследовании рассматривалась депрессия как индикатор субъективного неблагополучия.

Выборку эмпирического исследования составили сотрудники различных организаций крупного промышленного города чентральной части России (N = 104). На основе индикаторов субъективной негарантированности работы были выделены две подвыборки: 1) воспринимающие свою работу как негарантированную (44 сотрудника); 2) воспринимающие свою работу как относительно гарантированную (60 сотрудников). Далее в этих двух подгруппах сравнивались показатели временной перспективы и уровень депрессии, в результате были выявлены статистически значимые различия по отдельным временным ориентациям (на негативное прошлое и фаталистическое настоящее - более высокие значения в группе сотрудников, воспринимающих свою работу как негарантированную), а также по уровню депрессии (также более высокий уровень в группе с переживанием негарантированности). Результаты корреляционного анализа, проведенного на основе индикаторов 
негарантированной работы, показателей временной перспективы и уровня депрессии, косвенно подтвердили выявленные различия в двух подвыборках. Дисперсионный анализ показал, что изменения временной перспективы, а также субъективное восприятие своей работы как негарантированной вносят определенный вклад в повышение депрессивного фона у таких сотрудников, что, в свою очередь, усиливает восприятие своего положения на работе как ненадежного.

Ключевые слова: негарантированная занятость, негарантированная работа, безработица, временная перспектива, негативное прошлое, фаталистическое настоящее, ориентация на будущее, депрессия, психологическая помощь, тренинговые технологии.

Для цитирования: Чуйкова Т. С., Сотникова Д. И. Временная перспектива в условиях негарантированной работы // Российский психологический журнал. - 2017. - Т. 14. - № 1. - С. 188-213.

Материалы статьи получены 13.07.2016

UDC 159.9:331

doi: 10.21702/rpj.2017.1.12

\title{
TIME PERSPECTIVE IN THE CONTEXT OF THE UNGUARANTEED WORK
}

\author{
Tatiana S. Chuykova ${ }^{1 *}$, Dar'ya I. Sotnikova ${ }^{2}$ \\ ${ }^{1}$ Akmulla Bashkir State Pedagogical University, Ufa, Russian Federation \\ 2 Uralsib Bank, Ufa, Russian Federation \\ * Correspondence author. E-mail: cts1004@bspu.ru
}

\section{Acknowledgments}

Supported by the Russian Humanitarian Science Foundation and the Academy of Sciences of the Republic of Bashkortostan, project no. 15-16-02017 "Relation to work in the context of the unguaranteed employment"

The paper examines features of employees' time perspective in the conditions of the unguaranteed work. Proceeding from the studies revealing time perspective distortion in the unemployed, the hypothesis states that similar effects can be characteristic for the unguaranteed work. The analysis of research on time perspective suggests that little attention has been devoted to studying this issue. However, a person experiences the uncertainty of the present and the future in a situation of the unguaranteed work; this involves a change in time perspective. Depression as an 
indicator of subjective trouble is another important consequence of the experience of the unguaranteed work.

The empirical study involved employees of various organizations of a large industrial city in the central part of Russia $(n=104)$. On the basis of subjective parameters of the unguaranteed work, the sample was split into two subsamples: (a) the respondents perceiving their work as unguaranteed (44 employees); (b) the respondents perceiving their work as relatively guaranteed (60 employees). Next, the rates of time perspective and the level of depression were compared in these two subgroups. The findings revealed statistically significant differences between temporal orientations and the level of depression. The scores of the orientation towards the negative past and the fatalistic present, as well as the level of depression were higher in the group of employees perceiving their work as unguaranteed. The correlation analysis of the unguaranteed work parameters, time perspective scores, and the level of depression indirectly confirmed the differences between two subsamples. Variance analysis showed that changes in time perspective and subjective perceiving work as unguaranteed contributed to increasing depression among employees perceiving their own work as unguaranteed. In its turn, increasing depression may heighten the perception of their own working status as insecure.

Keywords: unguaranteed employment, unguaranteed work, unemployment, time perspective, past negative, present fatalistic, orientation towards future, depression, psychological assistance, training techniques.

For citation: Chuykova T. S., Sotnikova D. I. Time perspective in the context of the unguaranteed work. Rossiiskii psikhologicheskii zhurnal - Russian Psychological Journal, 2017, V. 14, no. 1, pp. 188-213 (in Russian).

Original manuscript received 13.07.2016

\section{Введение}

В современных социально-экономических условиях, характеризующихся выраженными изменениями рынка труда, сопряженными с процессами безработицы, отсутствием гарантий сохранения трудовой занятости для тех, кто ее имеет, чрезвычайно востребованными становятся исследования, направленные на изучение того, как эти изменившиеся реалии жизни влияют на психологическое состояние человека, и как можно ему помочь реагировать более конструктивно в обозначенных условиях с помощью технологий психологического сопровождения.

Достаточно развернуто подобные исследования представлены по отношению к ситуации вынужденной безработицы, их результаты указывают на значительные изменения психологического и социально-психологического 
статуса человека, оказавшегося в подобной ситуации. Ухудшение материального положения, потеря социальных связей, поддерживаемых благодаря работе, размывание социального статуса, изменение личностной идентичности - это далеко не полный перечень последствий безработицы, описанных по результатам исследований различными авторами (см. обзор в [19]). В исследованиях, посвященных проблематике психологических последствий безработицы, также фиксируются изменения восприятия времени [8, 33], которые могут обусловливать негативные изменения в психическом здоровье и различные девиации поведения у безработных. М. Яхода делает акцент на неспособности безработных заполнять повседневное время, структурировать его, т. е. локализует эти изменения в подструктуре настоящего. Кроме того, в результате потери связи безработного с производственным коллективом и прекращения участия в решении коллективных целей, происходят закономерные изменения, проявляющиеся в обеднении будущего [33]. Безработные чаще других склонны воспринимать свое будущее как неопределенное и часто в негативном аспекте [27]. По результатам исследования Е. Ю. Мандриковой [8], безработные ощущают себя психологически старше своего реального возраста, они в большей степени ориентированы на настоящее, чем на другие векторы своего субъективного времени, для них характерно неверие в свои способности контролировать события собственной жизни.

Результаты наших исследований $[20,24]$ также выявили искажение временной перспективы у безработных. Мы зафиксировали более чем у половины безработных, вошедших в выборку исследования, частичную или полную фрустрацию базовых потребностей в настоящем, что дало нам основания полагать, что текущий период их жизни воспринимался ими как обедненный смыслами, ценностями, событиями.

В соответствии с этими результатами мы реализовывали в группах безработных тренинговые технологии, направленные, в том числе, на коррекцию у них субъективного восприятия времени, что имело позитивное влияние на эмоциональное состояние и стратегии поведения безработных. В ходе тренинговой работы с безработными мы пришли к пониманию того, что внутренние переживания, обусловленные потерей работы, можно уподобить некоторому обрыву в последовательном течении жизни, резкому нарушению устоявшегося жизненного ритма, «сцепленного» с прошлым и будущим. В этом и проявлялось у наших безработных нарушение временной перспективы, идущей из прошлого через настоящее в будущее. Внутренняя работа сознания направлялась на восстановление прерванной жизненной перспективы через фазы «увязания» в прошлом, его переоценки, затем попыток переструктурирования будущего и восстановления 
интенциональности и способности «моделировать будущее», что и приводило к воссозданию оборванной цепи, в которую логично встраивалось изменившееся настоящее. Тренер в такой группе выступал, в известном смысле, в качестве фасилитатора этих процессов, этому помогала и групповая динамика, направляемая им.

Содержательно наши усилия направлялись на актуализацию прошлого опыта участников группы, связанного с успешным разрешением трудных ситуаций и решением сложных задач, тем самым активизировались ресурсы субъектной активности, аккумулированные в их прошлом опыте. Мы также помогали участникам тренинговой группы формировать более отчетливую траекторию будущего, выстраивать планы и конкретные пути их достижения. Далеко не всегда это получалось в полной мере, но определенный импульс такой внутренней работе в группе задавался, а далее все зависело от самого безработного. Следует отметить, что результативность тренинговых технологий в коррекции субъективного восприятия времени у безработных была подтверждена и другими исследователями. Так, И. А. Волошина с соавторами отмечают, что при личностно-ориентированной направленности тренинговой работы удается уменьшить фиксацию безработных на переживании настоящей, неблагоприятной для них ситуации, а также помочь в осмыслении ими прошлого, настоящего и будущего в позитивной перспективе [2]. Полагаем, что без прохождения этого этапа, направленного на «выравнивание» временной перспективы, последующие шаги, связанные с отработкой навыков поиска работы в условиях тренинга и применением их в реальных ситуациях, будут эмоционально затратными и недостаточно эффективными.

\section{Анализ исследований временной перспективы}

В прикладных психологических исследованиях временная перспектива рассматривается как фактор психологического здоровья $[4,12,14,16]$, как мишень для коррекционной и развивающей работы с детьми и подростками [15]. Искажения психологического времени, проявляющиеся в утрате временной перспективы будущего, отмечаются у юношей с аддиктивным поведением $[16,17]$, а также у подростков, воспитывающихся в учреждении для детей-сирот и детей, оставшихся без попечения родителей: у них формируется «...другое, укороченное, будущее, другое, бедное воспоминаниями прошлое, другое, как будто бы и насыщенное актуальными заботами, целями, мотивами, но субъективно “еле ползущее" настоящее время» [16, с. 40].

У людей, переживающих трудную жизненную ситуацию, также отмечаются деформации временной трансспективы, - определяемой В. И. Ковалевым (1995) как способность обозрения человеком течения времени 
собственной жизни в любом его направлении, как возможность взаимосоотнесения прошлого, будущего и настоящего и связывания этих временных компонентов человеческой жизни в его сознании (см. [10]). Эти деформации описываются авторами следующим образом: «будущее для них утрачивает свою позитивную валентность, настоящее хаотично, а прошлое перестает быть ресурсом для продуктивного жизнетворчества» [10, с. 96]. Представлены и другие описания изменений временной перспективы в кризисной ситуации. Так, в исследованиях отмечаются в качестве таких следствий выраженная фиксация на прошлом [32], ограниченность и негативный характер ориентации на будущее [35].

В условиях постоянных изменений жизненных обстоятельств, ставших обычными для современной социальной действительности, наблюдаются отчетливые изменения временной перспективы, что зафиксировано в исследованиях, проведенных в России периода распада Советского Союза, и в особенности после дефолта 1998 г. Так, по результатам исследования К. Муздыбаева [9] временная перспектива у большей части респондентов в тот период характеризовалась следующими особенностями: у значительного количества опрошенных превалировала ориентация на прошлое, при этом настоящее воспринималось исключительно в негативном ключе, а прошлое - в позитивном.

В то же время отечественные исследователи временной перспективы [7] отмечают, что в настоящее время фокус внимания исследователей в области временной перспективы направлен на изучение связи этого конструкта с другими характеристиками индивидуальных различий. В качестве примера можно привести исследование временной перспективы у студентов, характеризующихся выраженной прокрастинацией [3]. Исследования, рассматривающие временную перспективу в более широком социальном контексте, пока не многочисленны, хотя особенно востребованы на фоне тех потрясений и изменений, которые российское общество переживает в последние десятилетия, и которые не могли не повлиять на отношение людей к разным периодам времени их жизни. К подобного рода кардинальным изменениям, фиксируемым в сфере занятости, но оказывающим сильное воздействие на все сферы жизни человека, с полным правом можно отнести безработицу и отсутствие гарантий занятости для работающих людей. О негативных изменениях в восприятии временной перспективы у безработных, зафиксированных в исследованиях как зарубежных, так и отечественных авторов, мы писали выше. Полагаем, что подобного рода изменения могут происходить не только в ситуации потери работы, но и при восприятии человеком подобной угрозы, т. е. в ситуации негарантированной работы, которая становится все более характерной для нашего времени. 


\section{О психологическом феномене «негарантированная работа»}

Исследования негарантированной работы представлены в зарубежной литературе, начиная с 1980-х годов. Л. Гринхалт и 3. Розенблат (Greenhalgh, Rosenblatt) одни из первых предложили определение рассматриваемому феномену, описав его следующим образом: «...беспомощность человека в сохранении работы в неблагоприятной ситуации» [28, с. 365]. К настоящему времени появились и другие определения, содержательно схожие с отмеченным выше (см. обзор в [18]), приведем здесь одно из них, наиболее полно, по нашему мнению, отражающее психологические особенности рассматриваемого феномена: «...субъективно воспринимаемая и нежелательная вероятность потерять работу в будущем, а также страх и беспокойство, связанные с этой возможностью потери работы» [28, с. 365]. Авторы называют основные характеристики феномена:

- во-первых, субъективность переживания негарантированной работы, которая является следствием индивидуально своеобразного восприятия разными людьми актуальной производственной ситуации. Часто можно наблюдать, как сходные условия на работе могут по-разному восприниматься и интерпретироваться работниками и вызывать различные реакции. Также и условия негарантированной занятости могут оцениваться людьми неоднозначно;

- во-вторых, негативный характер переживания в ситуации негарантированной работы. В этом смысле рассматриваемый феномен не распространяется на тех людей, которые в силу различных жизненных обстоятельств выбирают работу на временной основе;

- в-третьих, неопределенность будущего, рассматриваемая авторами в качестве основного ядра в переживании ситуации негарантированной работы [28] (см. также [25, 37]).

В своих исследованиях мы отграничиваем феномен негарантированной работы, рассматривая его как психологический по своей природе, от социально-экономического явления негарантированной занятости, отражающего современные процессы экономической глобализации. Эти процессы отмечены усиливающейся конкуренцией как на национальном, так и глобальном уровнях, постоянными реструктуризациями, проводимыми в организациях и компаниях, сопровождающимися уменьшением численности персонала и переходом к использованию временных контрактов. В результате этих процессов формируются условия негарантированной занятости, которые, в свою очередь, могут восприниматься конкретными людьми далеко не равнозначно в силу субъективности человеческого восприятия, а также индивидуально-специфической производственной ситуации каждого человека. Именно такое понимание закладывается нами при 
разведении терминов «негарантированная занятость» и «негарантированная работа»: если в первом случае речь идет об объективно складывающихся условиях экономической нестабильности и неопределенности, напрямую отражающихся на условиях трудовой занятости, то во втором - речь идет об индивидуально-своеобразном отражении таких условий разными людьми.

В зарубежных исследованиях зафиксированы негативные следствия переживания негарантированной работы, такие как снижение удовлетворенности работой, ослабление приверженности (лояльности) по отношению к организации, увеличение текучести кадров (см., напр. [31, 34, 38]). В качестве обобщающих эти и подобные им результаты могут послужить данные, представленные в метаисследовании, охватывающем 172 выборки с общей численностью 132927 сотрудников, работающих в организациях и компаниях различных стран. Корреляционный анализ, проведенный авторами исследования на столь обширной выборке, выявил значимые связи между негарантированностью работы и производительностью труда $(r=-0,21)$, доверием к организации $(r=-0,49)$, психологическим $(r=-0,28)$ и физическим здоровьем $(r=-0,23)$ [23].

По результатам исследований, в качестве следствий негарантированной работы могут выступать увеличение заболеваемости, выявляемое как на основании самоотчетов, так и по объективным показателям, рост депрессии и тревоги [30,36], психосоматические нарушения, такие как расстройства сна, головокружения, потеря аппетита [21]. Некоторые авторы отмечают негативный эффект негарантированной работы на состояние здоровья работника, соотносимый по своей выраженности с безработицей [22].

В изученной нами литературе, посвященной феномену негарантированной работы, мы не обнаружили исследований, в которых бы непосредственно фиксировались искажения временной перспективы у людей, работающих в подобных условиях. Они скорее подразумеваются исходя из трактовок данного феномена, ядерным аспектом которого большинством авторов называется неопределенность в отношении будущего. Мы предполагаем, что в условиях негарантированной работы у человека изменяются в той или иной степени все аспекты временной перспективы: восприятие прошлого, настоящего и будущего. Кроме того, мы полагаем, что эти изменения временной перспективы могут вносить весомый вклад в ухудшение психологического и физического здоровья таких работников, зафиксированного в исследованиях, описанных выше.

\section{Выборка, гипотезы и методы исследования}

Наше исследование проводилось в период с марта по октябрь 2014 г. Уровень безработицы в России в тот период достиг минимальной 
отметки (5,2\%) в сравнении с показателем кризисного 2009 г., на который пришелся пик роста безработицы (8,3\%). Для сравнения: уровень безработицы на конец 2015 г. составлял уже 5,6\%, указывая на то, что кривая безработицы медленно «поползла» вверх [1]. В то же время, мы должны учитывать все более увеличивающуюся представленность непостоянной занятости во всем мире, в том числе в России. В соответствии с докладом Международной организации труда, в 2015 г. только четвертая часть всей рабочей силы в мире работала на условиях постоянных контрактов, в то время как подавляющая часть являлась самозанятой, работала неофициально или на условиях краткосрочных контрактов. В этом же докладе отмечалось, что «даже те, кто работают на постоянной основе, не обеспечены гарантиями сохранения своей занятости, т. к. организационные реструктуризации и сокращения штата стали обычным делом» [35].

Выборку исследования составили 104 человека, в возрастном диапазоне от 20 до 54 лет, из них 32 мужчины и 72 женщины, сотрудники различных организаций крупного промышленного города, расположенного в центральной части России. Целью исследования являлось изучение особенностей временной перспективы и эмоционального благополучия в условиях восприятия негарантированной работы. Опираясь на результаты исследований, проведенных в различных странах, мы предполагали обнаружить снижение уровня эмоционального благополучия у сотрудников, воспринимающих свою работу в качестве негарантированной. В качестве косвенного показателя эмоционального неблагополучия мы, вслед за другими авторами (см., например, [5]), рассматривали уровень выраженности депрессии. Как отмечалось выше, несмотря на то, что нами не были обнаружены исследования, в которых зафиксированы изменения временной перспективы в условиях негарантированной работы, но специфика переживаний в подобных условиях, описанная разными авторами и увязываемая с неопределенностью восприятия как настоящего, так и будущего, подвела нас к предположению об искажении основных аспектов временной перспективы у лиц, воспринимающих свою работу негарантированной. Также мы полагаем, что искажения временной перспективы у таких работников вносят, наряду с другими возможными факторами, негативный вклад в развитие у них депрессивных симптомов.

Таким образом, гипотезы исследования были сформулированы следующим образом:

Гunomeза 1. У сотрудников, воспринимающих свою работу как негарантированную, фиксируется более высокий уровень депрессии.

Гиnomeзa 2. У сотрудников, воспринимающих свою работу как негарантированную, отмечаются изменения во временной перспективе со стороны восприятия прошлого, настоящего, будущего. 
Гunomeза 3. Мы предполагаем, что определенный вклад в повышение уровня депрессии у лиц с восприятием своей работы как негарантированной вносят показатели временной перспективы.

\section{Методики}

Методика измерения ненадежности работы (А. Н. Дёмин). Автор при использовании термина «ненадежная работа» придерживается более широкого подхода, который представлен в литературе по рассматриваемой нами проблематике [5, 6, 22, 32]. В соответствии с данным подходом, помимо высокой вероятности потери работы рассматриваются потенциальные потери конкретных аспектов, характеризующих занятость, такие как: уменьшение объемов занятости, изменение оплаты труда, смена должностной позиции, возможности продвижения в должности и др. В то же время, в предложенной им методике фокус внимания направлен на восприятие вероятности потери работы, что согласуется с нашим подходом, который также обозначен в соответствующей литературе $[26,27,29]$ (см. также $[18,21])$. Методика включает в себя две шкалы по 14 пунктов в каждой. Первая шкала (шкала ненадежности будущего) направлена на выявление восприятия респондентом своего положения в организации в будущем, соответственно пункты шкалы формулируются как продолжения положения «Мое будущее в данной организации...». Примеры продолжений следующие: «вызывает уверенность», «непредсказуемое», «вызывает тревогу». Опрашиваемый может с какими-то пунктами согласиться, другие - отвергнуть, предусматривается также неопределенный ответ. При работе со второй шкалой (ненадежности настоящего) предлагается по аналогии с первой ответить на вопрос «Сейчас мое положение в организации...», используя следующие продолжения: «вызывает растерянность», «удовлетворяет», «беспокоит меня», и др. А. Н. Дёмин, указывая на удовлетворительные показатели надежности и валидности методики, рекомендует ее к использованию в научных исследованиях [5]. Мы использовали показатели по двум шкалам методики в качестве субъективных индикаторов переживания негарантированной работы.

Индекс вероятных изменений на предприятии (И. А. Петрова). Методика направлена на определение уровня организационной неопределенности. В качестве индикаторов организационной неопределенности автором выделяются изменения в организации, которые могут прямо или косвенно указывать на возможные сокращения работников и часто воспринимаются как их предвестники: смена руководства, перепрофилирование производства, слияние с другой компанией, сокращение или увольнение части работников и др. Респонденты оценивают вероятность каждого из предложенных событий с помощью шкалы от 1 - «это практически исключено», до 5 - «это очень вероятно». 
В качестве показателя организационной неопределенности используется суммарный балл [11]. Мы использовали этот показатель в качестве косвенного индикатора субъективной негарантированности работы: чем он выше, тем более вероятно, что человек воспринимает свою работу как негарантированную. Показатель, получаемый по этой методике, существенно дополняет результаты первой методики и высоко коррелирует с ними.

Опросник В. Зунга (адаптация Т. И. Балашовой), направленный на измерение депрессии. Следует отметить, что данная методика получила широкое использование не только для фиксации депрессии, но и состояний, близких к ней, таких как сниженное настроение, наличие беспокойства. В этой связи сошлемся на исследование А. Д. Дёмина, использовавшего эту методику при изучении феномена ненадежной работы (аналог негарантированной работы). При этом он указывал на наличие научно подтвержденной положительной связи «...неопределенности социального, профессионального, материального статусов с психологическим неблагополучием, в частности, уровнем тревоги, эмоциональной подавленности и т. д.» [5, с. 73]. Мы также можем отметить использование различных измерительных инструментов для фиксации депрессии в исследованиях подобной тематики, проводимых зарубежными авторами $[28,30]$. В нашем исследовании мы рассматривали показатель по методике в качестве косвенного индикатора эмоционального неблагополучия.

Опросник временной перспективы Ф. Зимбардо (Zimbardo Time Perspective Inventory - ZTPI), в адаптации A. Сырцовой, Е. Т. Соколовой, О. В. Митиной, выявляет индивидуальные предпочтения и установки, связанные со временем. Опросник включает в себя 56 пунктов, которые оцениваются респондентами по 5-ти балльной шкале Ликерта. Пункты распределены по пяти шкалам: позитивное прошлое, негативное прошлое, гедонистическое настоящее, фаталистическое настоящее и будущее [13]. Таким образом, методика удовлетворяет задачам нашего исследования в том, что охватывает всю временную перспективу (прошлое, настоящее, будущее), причем с учетом различных эмоциональных валентностей (позитивной, негативной).

\section{Результаты исследования и их обсуждение}

На основе индикаторов субъективной негарантированности работы, в качестве которых в исследовании использовались индекс изменений на предприятии, неуверенность в настоящем в организации, неуверенность в будущем в организации, выборка была разделена на две группы: работники с относительно низкими значениями по обозначенным индикаторам составили группу «уверенных работников», таковых оказалось 60, и с относительно высокими значениями - группу «неуверенных работников», соответственно 
их насчитывалось 44 человека. Описательные статистические данные по этим двум группам, а также значения уровня значимости различий по критерию Манна - Уитни представлены в таблице 1.

Таблица 1.

Параметры неуверенности в настоящем и будущем в организации, индекса изменений на предприятии у сотрудников с разной выраженностью субъективной негарантированности работы

Table 1.

Uncertainty about the present ant the future in the organization and the index of changes in the organization among employees with various levels of subjective parameters of the unguaranteed work

\begin{tabular}{|c|c|c|c|c|c|}
\hline $\begin{array}{c}\text { Коли- } \\
\text { чество } \\
\text { человек } \\
\text { (всего } \\
104) \\
\text { Respon- } \\
\text { dents } \\
(n=104)\end{array}$ & $\begin{array}{l}\text { Гpynnbl } \\
\text { Groups }\end{array}$ & $\begin{array}{c}\text { Onисательная } \\
\text { cmamuстика } \\
\text { Descriptive } \\
\text { statistics }\end{array}$ & $\begin{array}{l}\text { Неуверенность } \\
\text { в настоящем } \\
\text { в организации } \\
\text { Uncertainty } \\
\text { about the } \\
\text { present in the } \\
\text { organization }\end{array}$ & $\begin{array}{l}\text { Неуверен- } \\
\text { ность } \\
\text { в будущем } \\
\text { в органи- } \\
\text { зации } \\
\text { Uncertainty } \\
\text { about the } \\
\text { future in } \\
\text { the organi- } \\
\text { zation }\end{array}$ & $\begin{array}{c}\text { Индекс из- } \\
\text { менений } \\
\text { на предпри- } \\
\text { ятuи } \\
\text { Index of } \\
\text { changes } \\
\text { in the } \\
\text { organization }\end{array}$ \\
\hline \multirow{3}{*}{60} & \multirow{3}{*}{$\begin{array}{l}\text { Уверенные } \\
\text { работники } \\
\text { Confident } \\
\text { employees }\end{array}$} & $\begin{array}{l}\text { Среднее } \\
\text { Average }\end{array}$ & 0.338 & 0.531 & 30.616 \\
\hline & & $\begin{array}{l}\text { Медиана } \\
\text { Median }\end{array}$ & 0.269 & 0.400 & 30.000 \\
\hline & & $\begin{array}{c}\text { CKO } \\
\text { Standard } \\
\text { deviation }\end{array}$ & 0.307 & 0.486 & 7.724 \\
\hline \multirow{3}{*}{44} & \multirow{3}{*}{$\begin{array}{l}\text { Неуве- } \\
\text { ренные } \\
\text { работники } \\
\text { Uncon- } \\
\text { fident } \\
\text { employees }\end{array}$} & $\begin{array}{l}\text { Среднее } \\
\text { Average }\end{array}$ & 1.438 & 1.313 & 39.409 \\
\hline & & $\begin{array}{l}\text { Медиана } \\
\text { Median }\end{array}$ & 1.384 & 1.333 & 40.500 \\
\hline & & $\begin{array}{c}\text { CKO } \\
\text { Standard } \\
\text { deviation }\end{array}$ & 0.331 & 0.465 & 9.672 \\
\hline \multicolumn{3}{|c|}{$\begin{array}{c}\text { p (для различий между группами) } \\
\text { Differences between groups }\end{array}$} & 0.000 & 0.000 & 0.000 \\
\hline
\end{tabular}

Далее для этих двух групп были сопоставлены значения показателей временной перспективы и уровня депрессии также с применением критерия Манна - Уитни (данные представлены в таблице 2). 
Таблица 2.

Параметры временной перспективы и депрессии у работников с разной выраженностью субъективной негарантированности работы

Table 2.

Time perspective and depression in employees with various levels of subjective parameters of the unguaranteed work

\begin{tabular}{|c|c|c|c|c|c|c|c|c|}
\hline $\begin{array}{c}\text { Коли- } \\
\text { чество } \\
\text { человек } \\
\text { (всего } \\
\text { 104) } \\
\text { Respon- } \\
\text { dents } \\
(n= \\
104)\end{array}$ & $\begin{array}{l}\text { Гpynnы } \\
\text { Groups }\end{array}$ & $\begin{array}{c}\text { Onиса- } \\
\text { тельная } \\
\text { статис- } \\
\text { тика } \\
\text { Descrip- } \\
\text { tive } \\
\text { statistics }\end{array}$ & $\begin{array}{c}\text { Неza- } \\
\text { тивное } \\
\text { прош- } \\
\text { лое } \\
\text { Past } \\
\text { Negative }\end{array}$ & $\begin{array}{c}\text { Гедо- } \\
\text { нисти- } \\
\text { ческое } \\
\text { настоя- } \\
\text { uee } \\
\text { Present } \\
\text { Hedo- } \\
\text { nistic }\end{array}$ & $\begin{array}{l}\text { Будy- } \\
\text { uee } \\
\text { Future }\end{array}$ & $\begin{array}{c}\text { Пози- } \\
\text { тивное } \\
\text { прош- } \\
\text { лое } \\
\text { Past } \\
\text { Positive }\end{array}$ & $\begin{array}{c}\text { Фата- } \\
\text { листи- } \\
\text { ческое } \\
\text { настоя- } \\
\text { щеe } \\
\text { Present } \\
\text { Fatalistic }\end{array}$ & $\begin{array}{l}\text { Уровень } \\
\text { деnрес- } \\
\text { cuu } \\
\text { Level of } \\
\text { depres- } \\
\text { sion }\end{array}$ \\
\hline \multirow{3}{*}{60} & \multirow{3}{*}{$\begin{array}{l}\text { Уверен- } \\
\text { ные ра- } \\
\text { ботники } \\
\text { Con- } \\
\text { fident } \\
\text { em- } \\
\text { ployees }\end{array}$} & $\begin{array}{r}\text { Среднее } \\
\text { Average }\end{array}$ & 2.421 & 3.073 & 3.979 & 4.062 & 2.448 & 33.050 \\
\hline & & $\begin{array}{c}\text { Медиана } \\
\text { Median }\end{array}$ & 2.272 & 3.000 & 4.076 & 4.111 & 2.333 & 32.000 \\
\hline & & $\begin{array}{c}\text { CKO } \\
\text { Standard } \\
\text { deviation }\end{array}$ & 0.723 & 0.731 & 0.663 & 0.648 & 0.746 & 8.679 \\
\hline \multirow{3}{*}{44} & \multirow{3}{*}{$\begin{array}{c}\text { Не- } \\
\text { уверен- } \\
\text { ные ра- } \\
\text { ботники } \\
\text { Unconfi- } \\
\text { dent em- } \\
\text { ployees }\end{array}$} & $\begin{array}{l}\text { Среднее } \\
\text { Average }\end{array}$ & 2.880 & 3.197 & 3.678 & 3.883 & 3.030 & 42.727 \\
\hline & & $\begin{array}{c}\text { Медиана } \\
\text { Median }\end{array}$ & 2.818 & 3.352 & 3.769 & 4.111 & 3.222 & 44.000 \\
\hline & & $\begin{array}{c}\text { CKO } \\
\text { Standard } \\
\text { deviation }\end{array}$ & 0.870 & 0.660 & 0.801 & 0.832 & 0.761 & 8.269 \\
\hline \multicolumn{3}{|c|}{$\begin{array}{c}\text { Значение критерия } \\
\text { U Maнна - Уитни } \\
\text { Mann -Whitney U test }\end{array}$} & 905,50 & 1178,50 & 1032,50 & 1186,0 & 752,50 & 552,50 \\
\hline \multicolumn{3}{|c|}{$\begin{array}{c}\text { р (для различий между } \\
\text { группами) } \\
\text { Differences between groups }\end{array}$} & 0.006 & 0.353 & 0.058 & 0.381 & 0.000 & 0.000 \\
\hline
\end{tabular}


Таблица 3.

Корреляции шкал - индикаторов негарантированной работы со шкалами временной перспективы и депрессии (коэффициент корреляции Спирмена)

Table 3.

Parameters of the unguaranteed work by scales of time perspective and depression (Spearman correlation coefficient)

\begin{tabular}{|c|c|c|c|}
\hline $\begin{array}{r}\text { Шкалы времен } \\
u \text { деn } \\
\text { Scales of time } \\
\text { depı }\end{array}$ & $\begin{array}{l}\text { oŭ перспективы } \\
\text { peccuu } \\
\text { perspective and } \\
\text { ession }\end{array}$ & $\begin{array}{c}\text { Неуверенность } \\
\text { в настоящем } \\
\text { в организации } \\
\text { Uncertainty about } \\
\text { the present in the }\end{array}$ & $\begin{array}{c}\text { Неуверенность } \\
\text { в будущем } \\
\text { в организации } \\
\text { Uncertainty about } \\
\text { the future in the }\end{array}$ \\
\hline & $\begin{array}{l}\text { Восприятие нега- } \\
\text { тивного прошлого } \\
\text { Perceiving Past } \\
\text { Negative }\end{array}$ & $0,325 * * *$ & $0,264^{*}$ \\
\hline & $\begin{array}{l}\text { Восприятие пози- } \\
\text { тивного прошлого } \\
\text { Perceiving Past } \\
\text { Positive }\end{array}$ & $-0,134$ & $-0,089$ \\
\hline $\begin{array}{l}\text { Шкалы временной } \\
\text { ориентации } \\
\text { Scales of temporal } \\
\text { orientation }\end{array}$ & $\begin{array}{l}\text { Восприятие фа- } \\
\text { талистического } \\
\text { настоящего } \\
\text { Perceiving Present } \\
\text { Fatalistic }\end{array}$ & $0,369 * * *$ & $0,238^{*}$ \\
\hline & $\begin{array}{l}\text { Восприятие ге- } \\
\text { донистического } \\
\text { настоящего } \\
\text { Perceiving Present } \\
\text { Hedonistic }\end{array}$ & 0,034 & 0,022 \\
\hline & $\begin{array}{l}\text { Ориентация } \\
\text { на будущее } \\
\text { Orientation towards } \\
\text { Future }\end{array}$ & $-0,154$ & $-0,013$ \\
\hline $\begin{array}{c}\text { Уровень } \\
\text { Level of }\end{array}$ & $\begin{array}{l}\text { депрессии } \\
\text { depression }\end{array}$ & $0,574 * * *$ & $0,363 * * *$ \\
\hline
\end{tabular}

Примечание: ${ }^{*}-p<0,05 ;{ }^{* *}-p<0,01,{ }^{* * *}-p<0,001$.

Note: ${ }^{*}-p<0.05$; $^{*}-p<0.01,{ }^{* * *}-p<0.001$. 
Как видим из таблицы 2, для группы «неуверенных» работников показатели ориентаций на негативное прошлое и фаталистическое настоящее, а также уровень депрессии статистически значимо выше, чем в группе «уверенных» работников. По другим временным ориентациям различий между группами не выявлено. Обнаруженные особенности подтверждают и результаты корреляционного анализа индикаторов негарантированной работы и показателей временной перспективы и депрессии (см. таблицу 3).

Таким образом, основываясь на результатах применения критерия Манна - Уитни и с учетом данных корреляционного анализа, мы можем отметить, что у сотрудников, воспринимающих свою работу как негарантированную, фиксируется более высокий уровень депрессии, что подтверждает гunomeзу 1. Также у данной группы сотрудников фиксируется повышение ориентации на негативное прошлое и фаталистическое настоящее, что частично подтверждает гипотезу 2.

Для проверки гипотезы 3 о влиянии временной перспективы (ее различных аспектов) на уровень депрессии мы применили метод дисперсионного анализа ANOVA.

Перед применением дисперсионного анализа мы проверили нормальность распределения соответствующих показателей путем расчета асимметрии и эксцесса этих показателей, и сопоставления их с критическими значениями (см. таблицу 4).

Если стандартная ошибка асимметрии и эксцесса более чем в 3 раза превышает абсолютное значение асимметрии и эксцесса, то гипотеза о нормальности распределения признака отклоняется. В нашем случае, показатель гедонистического настоящего не удовлетворяет нормальному распределению, т. к. ошибка асимметрии превышает абсолютное значение асимметрии в 9 раз. Поскольку значимой корреляции между показателем гедонистического настоящего и депрессией не было выявлено, исключение данного показателя из анализа не повлияет на проверку гипотезы. Следовательно, в дисперсионном анализе показатель гедонистического настоящего участвовать не будет.

На первом этапе анализа, в качестве зависимой переменной выступил показатель депрессии, независимых переменных - отдельные временные ориентации и психологические индикаторы негарантированной работы. В результате мы выявили весомый вклад в развитие депрессивной симптоматики неуверенности в настоящем ( $F=15,541 ; p=0,000)$ и будущем $(F=12,906$; $p=0,000)$ в организации, и более умеренный со стороны временных ориентаций на позитивное прошлое $(F=1,862 ; p=0,041)$ и фаталистическое настоящее ( $F=2,157 ; p=0,012)$. Данные дисперсионного анализа представлены в таблице 5 . 
Таблица 4.

Описательная статистика показателей временной перспективы, депрессии и отношения к работе

Table 4.

Descriptive statistics of time perspective, depression, and relation to work

\begin{tabular}{|c|c|c|c|c|c|c|}
\hline $\begin{array}{c}\text { Описательная } \\
\text { статистика психоло- } \\
\text { гических показателей } \\
\text { Descriptive statistics } \\
\text { of psychological } \\
\text { parameters }\end{array}$ & $\begin{array}{c}\text { Среднее } \\
\text { зна- } \\
\text { чение } \\
\text { Mean } \\
\text { score }\end{array}$ & $\begin{array}{c}\text { Meдиана } \\
\text { Median }\end{array}$ & $\begin{array}{c}\text { Aсим- } \\
\text { mempuя } \\
\text { Skewness }\end{array}$ & $\begin{array}{l}\text { Ошибка } \\
\text { асимме- } \\
\text { mpuu } \\
\text { Error of } \\
\text { skewness }\end{array}$ & $\begin{array}{l}\text { Эксиесс } \\
\text { Kurtosis }\end{array}$ & $\begin{array}{r}\text { Ошибка } \\
\text { эксцесса } \\
\text { Error of } \\
\text { kurtosis }\end{array}$ \\
\hline $\begin{array}{l}\text { Уровень депрессии } \\
\text { Level of depression }\end{array}$ & 34,336 & 33,000 & 0,429 & 0,236 & $-0,320$ & 0,469 \\
\hline $\begin{array}{l}\text { Негативное прошлое } \\
\text { Past Negative }\end{array}$ & 2,615 & 2,454 & 0,582 & 0,236 & $-0,386$ & 0,469 \\
\hline $\begin{array}{l}\text { Гедонистическое } \\
\text { настоящее } \\
\text { Present Hedonistic }\end{array}$ & 3,126 & 3,117 & 0,025 & 0,236 & $-0,529$ & 0,469 \\
\hline $\begin{array}{l}\text { Будущее } \\
\text { Future }\end{array}$ & 3,852 & 3,923 & $-0,351$ & 0,236 & $-0,713$ & 0,469 \\
\hline $\begin{array}{l}\text { Позитивное прошлое } \\
\text { Past Positive }\end{array}$ & 3,987 & 4,111 & $-0,844$ & 0,236 & 0,399 & 0,469 \\
\hline $\begin{array}{l}\text { Фаталистическое } \\
\text { настоящее } \\
\text { Present Fatalistic }\end{array}$ & 2,694 & 2,777 & 0,086 & 0,236 & $-0,771$ & 0,469 \\
\hline $\begin{array}{l}\text { Неуверенность в буду- } \\
\text { щем в организации } \\
\text { Uncertainty about } \\
\text { the future in the } \\
\text { organization }\end{array}$ & 0,862 & 0,833 & 0,185 & 0,236 & $-1,079$ & 0,469 \\
\hline $\begin{array}{l}\text { Неуверенность в насто- } \\
\text { ящем в организации } \\
\text { Uncertainty about } \\
\text { the present in the } \\
\text { organization }\end{array}$ & 0,803 & 0,769 & 0,267 & 0,236 & $-1,108$ & 0,469 \\
\hline $\begin{array}{l}\text { Индекс изменений } \\
\text { в организации } \\
\text { Index of changes in the } \\
\text { organization }\end{array}$ & 34,336 & 33,000 & 0,429 & 0,236 & $-0,320$ & 0,469 \\
\hline
\end{tabular}


Таблица 5.

\section{Влияние показателей субъективной негарантированности и временной перспективы на уровень депрессии (ANOVA)}

Table 5.

\section{Subjective parameters of the unguaranteed work and time perspective} by the level of depression (ANOVA)

\begin{tabular}{|c|c|c|}
\hline \multirow{2}{*}{$\begin{array}{c}\text { Влияние nсихологических показателей на уровень } \\
\text { деnрессии } \\
\text { Psychological parameters by the level of depression }\end{array}$} & \multicolumn{2}{|c|}{$\begin{array}{l}\text { Дenpeccuя } \\
\text { Depression }\end{array}$} \\
\hline & $F$ & $p$ \\
\hline $\begin{array}{l}\text { Неуверенность в настоящем в организации } \\
\text { Uncertainty about the present in the organization }\end{array}$ & 15,541 & 0,000 \\
\hline $\begin{array}{l}\text { Неуверенность в будущем в организации } \\
\text { Uncertainty about the future in the organization }\end{array}$ & 12,906 & 0,000 \\
\hline $\begin{array}{l}\text { Индекс изменений в организации } \\
\text { Index of changes in the organization }\end{array}$ & 1,272 & 0,197 \\
\hline $\begin{array}{l}\text { Негативное прошлое } \\
\text { Past Negative }\end{array}$ & 1,714 & 0,058 \\
\hline $\begin{array}{l}\text { Позитивное прошлое } \\
\text { Past Positive }\end{array}$ & 1,862 & 0,041 \\
\hline $\begin{array}{l}\text { Фаталистическое настоящее } \\
\text { Present Fatalistic }\end{array}$ & 2,157 & 0,012 \\
\hline $\begin{array}{l}\text { Будущее } \\
\text { Future }\end{array}$ & 1,167 & 0,308 \\
\hline
\end{tabular}

Предположив также наличие встречного влияния депрессии на восприятие ситуации на работе и временные ориентации, мы провели повторный дисперсионный анализ, поменяв местами зависимую и независимые переменные. Соответственно, выделили в качестве независимой переменной уровень депрессии для определения меры его влияния на показатели субъективной негарантированности и временные ориентации. В таблице 6 представлены результаты данного анализа.

Уровень депрессии оказывает значимое влияние на неуверенность сотрудников в настоящем в организации, индекс изменений в организации, а также ориентацию на фаталистическое настоящее.

Исходя из полученных данных, можно сделать вывод о том, что неуверенность в настоящем и будущем в организации в большей степени влияют на уровень депрессии, чем депрессия на данные показатели. Однако уровень депрессии имеет большее влияние на индекс изменений на предприятии. Можно предположить, что тревога за свою работу приводит к упадку настроения у сотрудника, что, в свою очередь, заставляет его делать более пессимистичные прогнозы о положении дел в организации. Так, встревоженный сотрудник 
может больше обращать внимание на слухи относительно того, как обстоят дела в организации, которые могут и не иметь под собой оснований, строить догадки, тем самым еще больше усиливая свое беспокойство.

Таблица 6.

\section{Влияние уровня депрессии на показатели субъективной негарантированности и временной перспективы (ANOVA)}

Table 6.

Level of depression by subjective parameters of the unguaranteed work and time perspective (ANOVA)

\begin{tabular}{|c|c|c|}
\hline \multirow{2}{*}{$\begin{array}{l}\text { Влияние уровня депрессии на показатели негаран- } \\
\text { тированной занятости } \\
\text { Level of depression by unguaranteed employment } \\
\text { parameters }\end{array}$} & \multicolumn{2}{|c|}{$\begin{array}{l}\text { Уровень деnpeccuu } \\
\text { Level of Depression }\end{array}$} \\
\hline & $F$ & $p$ \\
\hline $\begin{array}{l}\text { Неуверенность в настоящем в организации } \\
\text { Uncertainty about the present in the organization }\end{array}$ & 2,1384 & 0,003 \\
\hline $\begin{array}{l}\text { Неуверенность в будущем в организации } \\
\text { Uncertainty about the future in the organization }\end{array}$ & 1,4151 & 0,110 \\
\hline $\begin{array}{l}\text { Индекс изменений в организации } \\
\text { Index of changes in the organization }\end{array}$ & 1,780 & 0,021 \\
\hline \multirow{2}{*}{$\begin{array}{l}\text { Влияние уровня депрессии на показатели временной } \\
\text { opuентации } \\
\text { Level of depression by temporal orientation parameters }\end{array}$} & \multicolumn{2}{|c|}{$\begin{array}{l}\text { Уровень denpeccuu } \\
\text { Level of Depression }\end{array}$} \\
\hline & $F$ & $p$ \\
\hline $\begin{array}{l}\text { Негативное прошлое } \\
\text { Past Negative }\end{array}$ & 1,315 & 0,166 \\
\hline $\begin{array}{l}\text { Позитивное прошлое } \\
\text { Past Positive }\end{array}$ & 1,483 & 0,082 \\
\hline $\begin{array}{l}\text { Фаталистическое настоящее } \\
\text { Present Fatalistic }\end{array}$ & 2,069 & 0,005 \\
\hline $\begin{array}{l}\text { Будущее } \\
\text { Future }\end{array}$ & 1,424 & 0,106 \\
\hline
\end{tabular}

Также мы можем сделать вывод о большем влиянии некоторых показателей временной перспективы на уровень депрессии. Показатель фаталистического настоящего влияет на уровень депрессии в большей мере. Показатель позитивного прошлого отрицательно влияет на уровень депрессии. Описанная динамика влияний представлена на рисунке 1. 


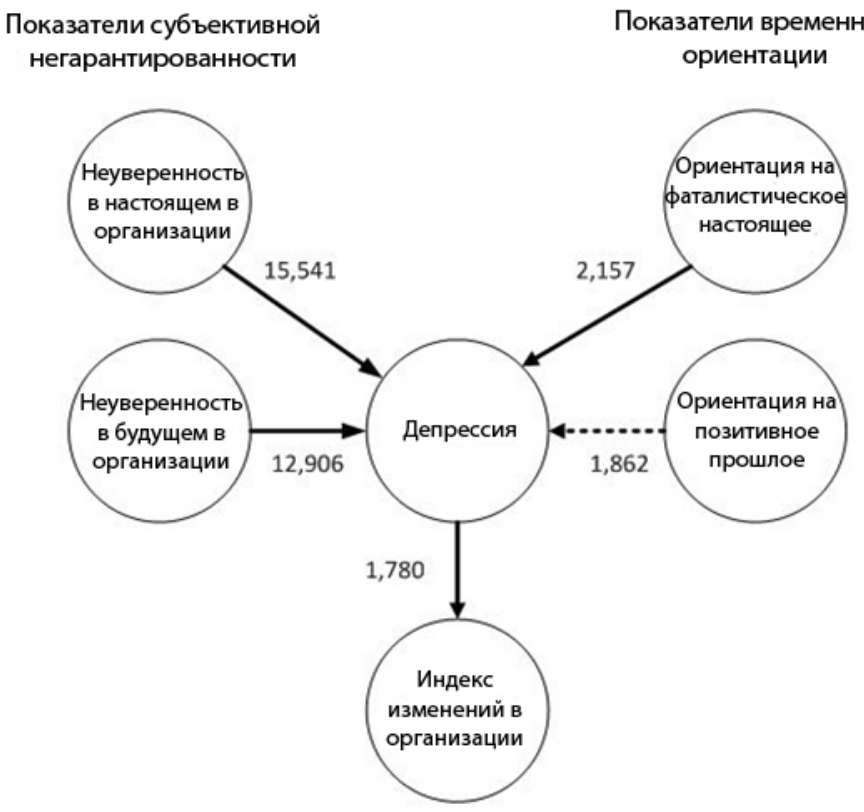

Рисунок 1. Влияние показателей субъективной негарантированности и временной перспективы на уровень депрессии

Условные обозначения: $\longrightarrow$ - положительная дисперсионная связь, $\rightarrow--\rightarrow$ - отрицательная дисперсионная связь.

Figure 1. Subjective parameters of the unguaranteed work and time perspective by the level of depression

Legend: $\longrightarrow$-positive dispersion, $\rightarrow-\rightarrow-$-negative dispersion.

Таким образом, результаты дисперсионного анализа свидетельствуют в пользу подтверждения гипотезы 3, и, кроме того, позволяют сформулировать более развернутый вывод. Субъективное восприятие работы как негарантированной (неуверенность в настоящем и будущем в организации), а также особенности временной перспективы, такие как ориентация на фаталистическое настоящее и низкий уровень ориентации на позитивное прошлое, влияют на эмоциональное состояние человека и ведут к проявлениям депрессии. Так, отсутствие у человека положительного опыта преодоления неудач в прошлом, незнание собственных сильных сторон, 
нежелание извлекать полезный урок из прошлых ситуаций (характерные для низкой ориентации на позитивное прошлое) и убеждение в том, что на ход жизни невозможно повлиять, сопровождаемое ленью, апатией и негативной реакцией на конкуренцию (характерные для ориентации на фаталистическое настоящее), ведут к депрессивному фону настроения. На этом фоне восприятие производственной ситуации как ненадежной еще больше усиливается. Таким образом, человек, работающий в условиях негарантированности, но при этом не верящий в свои силы и возможность повлиять на ситуацию, попадает в «ментальную ловушку», которая ведет к состояниям фрустрации и депрессии.

\section{Выводы}

На основе результатов проведенного исследования можно сделать следующие выводы:

- у лиц, воспринимающих свою работу как негарантированную, отмечается повышенный уровень депрессии, он не достигает клинического уровня, но указывает на состояния беспокойства, тревоги, разочарований;

- у лиц, воспринимающих свою работу как негарантированную, наблюдаются специфические особенности временной перспективы, которые проявляются в том, что они испытывают некоторое разочарование и недовольство своим прошлым и не рассматривают себя способными контролировать в должной мере события, происходящие с ними в настоящем;

- изменения временной перспективы со стороны прошлого и настоящего, отмеченные выше, а также субъективное восприятие своей работы как негарантированной вносят определенный вклад в повышение депрессивного фона у таких сотрудников, что, в свою очередь, усиливает восприятие своего положения на работе как ненадежного;

- при реализации различных технологий, связанных с оказанием психологической поддержки таким сотрудникам, следует учитывать эти изменения временной перспективы и проводить соответствующую их коррекцию. Подобного рода технологии разработаны, апробированы и подтвердили свою эффективность в тренинговой работе с безработными $[2,19]$ и могут быть использованы в психологическом сопровождении данной категории работников.

\section{Литература}

1. Безработица в России // URL: https://person-agency.ru/statistic.html

2. Волошина И. А., Галицына О. В., Гребенников В. А., Знакова Т. А. Групповая работа как форма психологической поддержки человека в ситуации безработицы // Вопросы психологии. - 1999. - № 4. - С. 43-51. 
3. Дементий Л. И., Карловская Н. Н. Особенности ответственности и временной перспективы у студентов с разным уровнем прокрастинации // Психология обучения. - 2013. - № 7. - С. 4-19.

4. Денисова Т. Н. Отношение ко времени у лиц с сохранной и нарушенной трудоспособностью: автореф. дисс. ... канд. психол. наук. М., 2002.

5. Дёмин А. Н. Психологическое измерение ненадежной работы // Человек. Сообщество. Управление. - 2008. - № 1. - С. 71-76.

6. Дёмин А. Н., Петрова И. А. Психологические эффекты угрозы потери работы // Психологический журнал. - 2010. - № 6. - С. 38-49.

7. Зарубин П. В., Сырцова А. Временная перспектива и экономическая нестабильность: сравнительное исследование 2007 и 2013 гг. // Психологические исследования. - 2013. - Т. 6.- № 32. - C. 9. - URL: http:// psystudy.ru

8. Мандрикова Е. Ю. Особенности психологического времени безработных // Вопросы психологии. - 2005. - № 6. - С. 54-62.

9. Муздыбаев К. Переживание времени в момент кризисов // Психологический журнал. - 2000. - № 4 (21). - С. 5-21.

10. Нуркова В. В., Василевская К. Н. Автобиографическая память в трудной жизненной ситуации: новые феномены // Вопросы психологии. - 2003. № 5. - С. 93-102.

11. Петрова И. А. Психологические эффекты пребывания личности в неопределенной жизненной ситуации (на примере ситуации ненадежной работы): дисс. ... канд. психол. наук. - Краснодар, 2011.

12. Спиридонова И. А. Временная трансспекция как методический прием при исследовании разных форм патологии // Методы психологии. Ежегодник РПО. - Ростов н/Д., 1997. - Т. 3. - Вып. 2. - С. 212-214.

13. Сырцова А., Соколова Е. Т., Митина О. В. Адаптация опросника временной перспективы личности Ф. Зимбардо // Психологический журнал. 2008. - T. 29. - № 3. - С. 101-109.

14. Толстых Н. Н. Временная перспектива и психическое здоровье // Психическое здоровье детей и подростков в контексте психологической службы / Под ред. И. В. Дубровиной. - М.: Издательский центр «Академия», 1997. - С. 146-152.

15. Толстых Н. Н. Некоторые задачи развития и коррекции временной перспективы у старших школьников // Практическая психология образования. Материалы I съезда практических психологов образования России. - Часть І. - М.: Изд-во РАО, 1994. - С. 92-96.

16. Толстых Н. Н. Развитие временной перспективы личности: культурноисторический подход: автореф. дисс. ... д. психол. наук. - М., 2010. 
17. Хомик В. С., Кроник А. А. Отношение к времени: психологические проблемы ранней алкоголизации и отклоняющегося поведения // Вопросы психологии. - 1988. - № 1. - С. 98-106.

18. Чуйкова Т. С. Негарантированная работа как социально-психологический феномен // Социальная психология и общество. - 2015. - Т. 6 - № 4. C. 139-149. doi:10.17759/sps.2015060410

19. Чуйкова Т. С. Психологические последствия безработицы: обзор исследований зарубежных авторов // Педагогический журнал Башкортостана. - 2011. - № 5 (36). - С. 254-262.

20. Чуйкова Т. С. Ценность работы в современных условиях трудовой занятости. - Уфа: Изд-во БГПУ, 2015. - 176 с.

21. Чуйкова Т. С., Сотникова Д. И. Особенности отношения к работе в условиях негарантированной занятости // Организационная психология.2016. - T. 6. - № 1. - C. 6-19. - URL: http://orgpsyjournal.hse.ru

22. Ashford S. J., Lee C., Bobko P. Content, causes, and consequences of job insecurity: A theory-based measure and substantive test // Academy of Management Journal. - 1989. - V. 32. - no. 4. - pp. 803-829.

23. Broom D. H., D'Souza R. M., Strazdins L., Butterworth P., Parslow R., Rodgers $B$. The lesser evil: Bad jobs or unemployment? A survey of mid-aged Australians // Social Science \& Medicine. - 2006. - V. 63. - pp. 575-586.

24. Cheng G. H. L., Chan D. K. S. Who suffers more from job insecurity? A metaanalytic review // Applied Psychology: An International Review. - 2008. V. 57. - no. 2. - pp. 272-303.

25. Chuikova T., Kurunov V. On the using of psychosemantic methods for the training practice with groups of the unemployed // Bulgarian Journal of Psychology. - 2010. - no. 1-4. - pp. 299-303.

26. Davis $M$. The effects of unemployment and poverty on sexual appetite and sexual risk in emerging and young adults // Sexual Addiction \& Compulsivity. - 2009. - V. 16 (4). - pp. 267-288.

27. De Witte H. Job insecurity and psychological well-being: Review of the literature and exploration of some unresolved issues // European Journal of Work and Organizational Psychology. - 1999. - V. 8. - no. 2. - pp. 155-177.

28. D'Souza R. M., Strazdins L., Lim L. L-Y., Broom D. H., Rodgers B. Work and health in a contemporary society: demands, control, and insecurity // Epidemial Community Health. - 2003. - V. 57. - pp. 849-854.

29. Elst T. V., De Witte H. \& De Cuyper N. The Job Insecurity Scale: A psychometric evaluation across five European countries // European Journal of Work and Organizational Psychology. - 2014. - no. 3. - pp. 364-380.

30. Ferrie J. E. Is job insecurity harmful to health? // Journal of the Royal Society of Medicine. - 2001. - V. 94. - pp. 47-79. 
31. Ferrie J. E., Shipley M. J., Stansfeld S. A., Marmot M. G. Effects of chronic job insecurity and change in job security on self reported health, minor psychiatric morbidity, physiological measures, and health related behaviours in British civil servants: the Whitehall II study // J. Epidemiol. Community Health. - 2002. - V. 56. - pp. 450-454.

32. Hellgren J., Sverke M. Does job insecurity lead to impaired well-being or vice versa? Estimation of cross-lagged effects using latent variable modeling // Journal of Organizational Behavior. - 2003. - V. 24. - pp. 215-236.

33. Holman E. A., Silver R. C. Getting "stuck" in the past: temporal orientation and coping with trauma // Journal of Personality and Social Psychology.1998. - V. 74 (5). - pp. 1146-1163.

34. Jahoda M., Lazarsfeld P. F., Zeisal H. Marienthal:The Sociology of an Unemployed Community. - New York: Aldine-Atherton, 1971.

35. Lam J. Job insecurity, adaptive strategies, and health in early adulthood (Order No. 3728173) // ProQuest Dissertations \& Theses A\&I (1734034367). - 2015. URL: http://search.proquest.com/docview/1734034367?accountid=175046

36. Lavi T., Solomon Z. Palestinian youth of the Intifada: PTSD and future orientation // Journal of the American Academy of Child and Adolescent Psychiatry. - 2005. - V. 44 (11). - pp. 1176-1183.

37. Rugulies R., Bultmann U., Aust B., Burr H. Psychosocial work environment and incidence of severe depressive symptoms: prospective findings from a 5-year follow-up of the Danish work environment cohort study // American Journal of Epidemiology. - 2006. - V. 163. - no. 10. - pp. 877-887.

38. Sverke M., Hellgren J., Naswal K. No security: A meta-analysis and review of job insecurity and its consequences // Journal of Occupational Health Psychology. - 2002. - no. 7. - pp. 242-264.

\section{References}

1. Unemployment in Russia. [Online]. (n. d.) Available at: https://person-agency. ru/statistic.html

2. Voloshina I. A., Galitsyna O. V., Grebennikov V. A., Znakova T. A. Group work as a form of psychological support to a person in a situation of unemployment. Voprosy psikhologii - Approaches to Psychology, 1999, no. 4, pp. 43-51 (in Russian).

3. Dementii L. I., Karlovskaya N. N. Responsibility and time perspective in students with various levels of procrastination. Psikhologiya obucheniya Psychology of Education, 2013, no. 7, pp. 4-19 (in Russian).

4. Denisova T. N. Otnoshenie ko vremeni u lits s sokhrannoi i narushennoi trudosposobnost'yu [Relation to time in employable and unemployable individuals]. Diss. Cand. Sci. (Psych.). Moscow, 2002. 
5. Demin A. N. A psychological dimension of unreliable work. Chelovek. Soobshchestvo. Upravlenie - Human. Community. Management, 2008, no. 1, pp. 71-76 (in Russian).

6. Demin A. N., Petrova I. A. Psychological effects of the threat of job loss. Psikhologicheskii zhurnal - Psychological Journal, 2010, no. 6, pp. 38-49 (in Russian).

7. Zarubin P. V., Syrtsova A. Time perspective and economic instability: a comparative study in 2007 and 2013. Psychological Studies - Psikhologicheskie Issledovaniya, 2013, V. 6, no. 32, p. 9 (in Russian). Available at: http://psystudy.ru

8. Mandrikova E. Yu. Features of psychological time in the unemployed. Voprosy psikhologii - Approaches to Psychology, 2005, no. 6, pp. 54-62 (in Russian).

9. Muzdybaev K. The experience of time in crises. Psikhologicheskii zhurnal Psychological Journal, 2000, no. 4 (21), pp. 5-21 (in Russian).

10. Nurkova V. V., Vasilevskaya K. N. Autobiographical memory in tight situations: new phenomena. Voprosy psikhologii - Approaches to Psychology, 2003, no. 5, pp. 93-102 (in Russian).

11. Petrova I. A. Psikhologicheskie effekty prebyvaniya lichnosti v neopredelennoi zhiznennoi situatsii (na primere situatsii nenadezhnoi raboty) [Psychological effects of an uncertain situation (on the example of unreliable work)]. Diss. Cand. Sci. (Psych.). Krasnodar, 2011.

12. Spiridonova I. A. Time transpection as a methodological way in studying various pathologies. Metody psikhologii. Ezhegodnik RPO - Methods of psychology. RPS Yearbook, Rostov-on-Don, 1997, V. 3, no. 2, pp. 212-214 (in Russian).

13. Syrtsova A., Sokolova E. T., Mitina O. V. Modification of Zimbardo time perspective inventory. Psikhologicheskii zhurnal - Psychological Journal, 2008, V. 29, no. 3, pp. 101-109 (in Russian).

14. Tolstykh N. N. Time perspective and mental health. In: Psikhicheskoe zdorov'e detei i podrostkov $v$ kontekste psikhologicheskoi sluzhby [Mental health of children and adolescents in psychological services]. Moscow, Akademiya Publ., 1997, pp. 146-152.

15. Tolstykh N. N. Nekotorye zadachi razvitiya i korrektsii vremennoi perspektivy u starshikh shkol'nikov [Some objectives of the development and correction of time perspective in senior pupils]. Prakticheskaya psikhologiya obrazovaniya. Materialy Is"ezda prakticheskikh psikhologov obrazovaniya Rossii [Proc. the 1st Congress of Practical Educational Psychologists of Russia"Practical education psychology"]. Moscow, RAE Publ., Part l, 1994, pp. 92-96.

16. Tolstykh N. N. Razvitie vremennoi perspektivy lichnosti: kul'turno-istoricheskii podkhod [Development of time perspective of personality: a cultural and historical approach]. Diss. Dr. Sci. (Psych.). Moscow, 2010. 
17. Khomik V. S., Kronik A. A. Relationship to time: psychological problems of early alcoholization and deviant behavior. Voprosy psikhologii - Approaches to Psychology, 1988, no. 1, pp. 98-106 (in Russian).

18. Chuykova T. S. Unguaranteed work as a socio-psychological phenomenon. Sotsial'naya psikhologiya iobshchestvo - Social Psychology and Society, 2015, V. 6, no. 4, pp. 139-149 (in Russian). doi:10.17759/sps.2015060410

19. Chuykova T. S. Psychological consequences of unemployment: a review of the studies of foreign authors. Pedagogicheskii zhurnal Bashkortostana-Pedagogical Journal of Bashkortostan, 2011, no. 5 (36), pp. 254-262 (in Russian).

20. Chuykova T. S. Tsennost' raboty $v$ sovremennykh usloviyakh trudovoi zanyatosti [The value of working in modern conditions of employment]. Ufa, Bashkir State Pedagogical University, 2015. 176 p.

21. Chuykova T. S., Sotnikova D. I. Features of the relation to work in conditions of unguaranteed employment. Organizatsionnaya psikhologiya - Organizational Psychology, 2016, V. 6, no. 1, pp. 6-19 (in Russian). Available at: http://orgpsyjournal.hse.ru

22. Ashford S. J., Lee C., Bobko P. Content, causes, and consequences of job insecurity: A theory-based measure and substantive test. Academy of Management Journal, 1989, V. 32, no. 4, pp. 803-829.

23. Broom D. H., D'Souza R. M., Strazdins L., Butterworth P., Parslow R., Rodgers B. The lesser evil: Bad jobs or unemployment? A survey of mid-aged Australians. Social Science \& Medicine, 2006, V. 63, pp. 575-586.

24. Cheng G. H. L., Chan D. K. S. Who suffers more from job insecurity? A metaanalytic review. Applied Psychology: An International Review, 2008, V. 57, no. 2, pp. 272-303.

25. Chuikova T., Kurunov V. On the using of psychosemantic methods for the training practice with groups of the unemployed. Bulgarian Journal of Psychology, 2010, no. 1-4, pp. 299-303.

26. Davis M. The effects of unemployment and poverty on sexual appetite and sexual risk in emerging and young adults. Sexual Addiction \& Compulsivity, 2009, V. 16 (4), pp. 267-288.

27. De Witte H. Job insecurity and psychological well-being: Review of the literature and exploration of some unresolved issues. European Journal of Work and Organizational Psychology, 1999, V. 8, no. 2, pp. 155-177.

28. D'Souza R. M., Strazdins L., Lim L. L-Y., Broom D. H., Rodgers B. Work and health in a contemporary society: demands, control, and insecurity. Epidemial Community Health, 2003, V. 57, pp. 849-854.

29. Elst T. V., De Witte H. \& De Cuyper N. The Job Insecurity Scale: A psychometric evaluation across five European countries. European Journal of Work and Organizational Psychology, 2014, no. 3, pp. 364-380. 
30. Ferrie J. E. Is job insecurity harmful to health? Journal of the Royal Society of Medicine, 2001, V. 94, pp. 47-79.

31. Ferrie J. E., Shipley M. J., Stansfeld S. A., Marmot M. G. Effects of chronic job insecurity and change in job security on self reported health, minor psychiatric morbidity, physiological measures, and health related behaviours in British civil servants: the Whitehall II study. J. Epidemiol. Community Health, 2002, V. 56, pp. 450-454.

32. Hellgren J., Sverke M. Does job insecurity lead to impaired well-being or vice versa? Estimation of cross-lagged effects using latent variable modeling. Journal of Organizational Behavior, 2003, V. 24, pp. 215-236.

33. Holman E. A., Silver R. C. Getting "stuck" in the past: temporal orientation and coping with trauma. Journal of Personality and Social Psychology, 1998, V. 74 (5), pp. 1146-1163.

34. Jahoda M., Lazarsfeld P. F., Zeisal H. Marienthal: The Sociology of an Unemployed Community. New York, Aldine-Atherton, 1971.

35. Lam J. Job insecurity, adaptive strategies, and health in early adulthood (Order No. 3728173). ProQuest Dissertations \& Theses A\&l (1734034367), 2015. URL: http://search.proquest.com/docview/1734034367?accountid=175046

36. Lavi T., Solomon Z. Palestinian youth of the Intifada: PTSD and future orientation. Journal of the American Academy of Child and Adolescent Psychiatry, 2005, V. 44 (11), pp. 1176-1183.

37. Rugulies R., Bultmann U., Aust B., Burr H. Psychosocial work environment and incidence of severe depressive symptoms: prospective findings from a 5-year follow-up of the Danish work environment cohort study. American Journal of Epidemiology, 2006, V. 163, no. 10, pp. 877-887.

38. Sverke M., Hellgren J., Naswal K. No security: A meta-analysis and review of job insecurity and its consequences. Journal of Occupational Health Psychology, 2002, no. 7, pp. 242-264. 